\title{
An influenza prophylaxis clinic in a primary school: 24 hours from notification
}

\section{to protection}

\author{
Kathryn M. Weston ${ }^{\mathrm{A}, \mathrm{B}}$ and George Truman ${ }^{\mathrm{A}}$ \\ ${ }^{\mathrm{A} C e n t r e ~ f o r ~ P o p u l a t i o n ~ H e a l t h, ~ S y d n e y ~ W e s t ~ A r e a ~ H e a l t h ~ S e r v i c e ~}$ \\ ${ }^{\mathrm{B} C o r r e s p o n d i n g ~ a u t h o r . E m a i l: ~ w e s t o n k @ w a h s . n s w . g o v . a u ~}$
}

\begin{abstract}
A public health clinic was established to provide antiviral prophylaxis to school contacts during the pandemic (H1N1) 2009 influenza outbreak in NSW, Australia. Children $(n=74)$ and staff $(n=9)$ were provided with antiviral (oseltamivir) prophylaxis following exposure to a confirmed case of pandemic (H1N1) 2009 influenza. The success of the clinic included attention to infection control and quarantining of potentially infectious children and staff, attendance at the clinic of pharmacists to ensure accurate dispensing of suspension medication, availability of experienced public health staff at short notice, and provision of accurate information to staff, school children and families attending the clinic.
\end{abstract}

Public health units (PHUs) are often required to plan, organise and establish clinics within short timeframes in order to reduce the risk of infectious diseases spreading into the wider community. Although policy documents are available outlining how to set up such clinics, few reports describe their successful operation and outcomes in terms of disease prevention. This paper describes a successful public health clinic providing antiviral prophylaxis to a large number of contacts during the CONTAIN phase of the pandemic (H1N1) 2009 influenza outbreak in New South Wales (NSW), Australia. ${ }^{1}$

\section{Notification and case history}

At 3.30 pm on 10 June 2009, the PHU at Sydney West Area Health Service (SWAHS), was advised of a symptomatic contact of a confirmed case of pandemic (H1N1) 2009 influenza during routine follow-up with the case. The contact was a 10-year-old boy from western Sydney. His family was immediately advised to take him to Children's Hospital at Westmead (CHW) for assessment.
Later that evening, the child attended CHW, describing a 2-day history of cough. A nasopharyngeal swab was referred for testing. Public health investigation revealed that the child had attended school on 9 and 10 June and had been in contact with 75 children and nine staff members. The high number of contacts related to the school's team-teaching approach.

\section{Planning}

Guidelines for oseltamivir prophylaxis recommend commencing the antiviral drug within 2 days of close contact with an infected individual. ${ }^{2}$ If the child was confirmed positive for pandemic (H1N1) 2009 influenza, exposed schoolchildren and staff would need to commence prophylaxis the following day for optimal effect.

At this stage, Australia was in CONTAIN phase, and no transmission of the influenza virus had been observed among SWAHS residents. World Health Organization advice for management of contacts included antiviral prophylaxis for close contacts and closure of classes or whole year cohorts at schools with confirmed cases. ${ }^{3}$ At 10 pm on 10 June a decision was made to set up a clinic at the school the following day. Because the pathology result would not be available until after midday, the clinic was established and ready to commence if the result was positive.

\section{The clinic day}

On the morning of 11 June, the situation and plans for the day were outlined to staff from the school and the district education office. The staff posted information on the school's website and contacted families of the exposed children in the class. Families were asked to attend the school at the end of the school day, when antiviral prophylaxis would be provided for their children if the result was positive.

The Incident Command System was used for operation of the clinic. ${ }^{4}$ The clinic was considered a high priority for SWAHS Centre for Population Health $(\mathrm{CPH})$ and eighteen staff were deployed at short notice from the CPH school vaccination, infectious diseases surveillance and environmental health teams. Other staff contacted by PHU to assist were two CHW pharmacists, and one SWAHS community health worker. In addition, three staff from the NSW Health Centre for Health Protection assisted. Clinic 

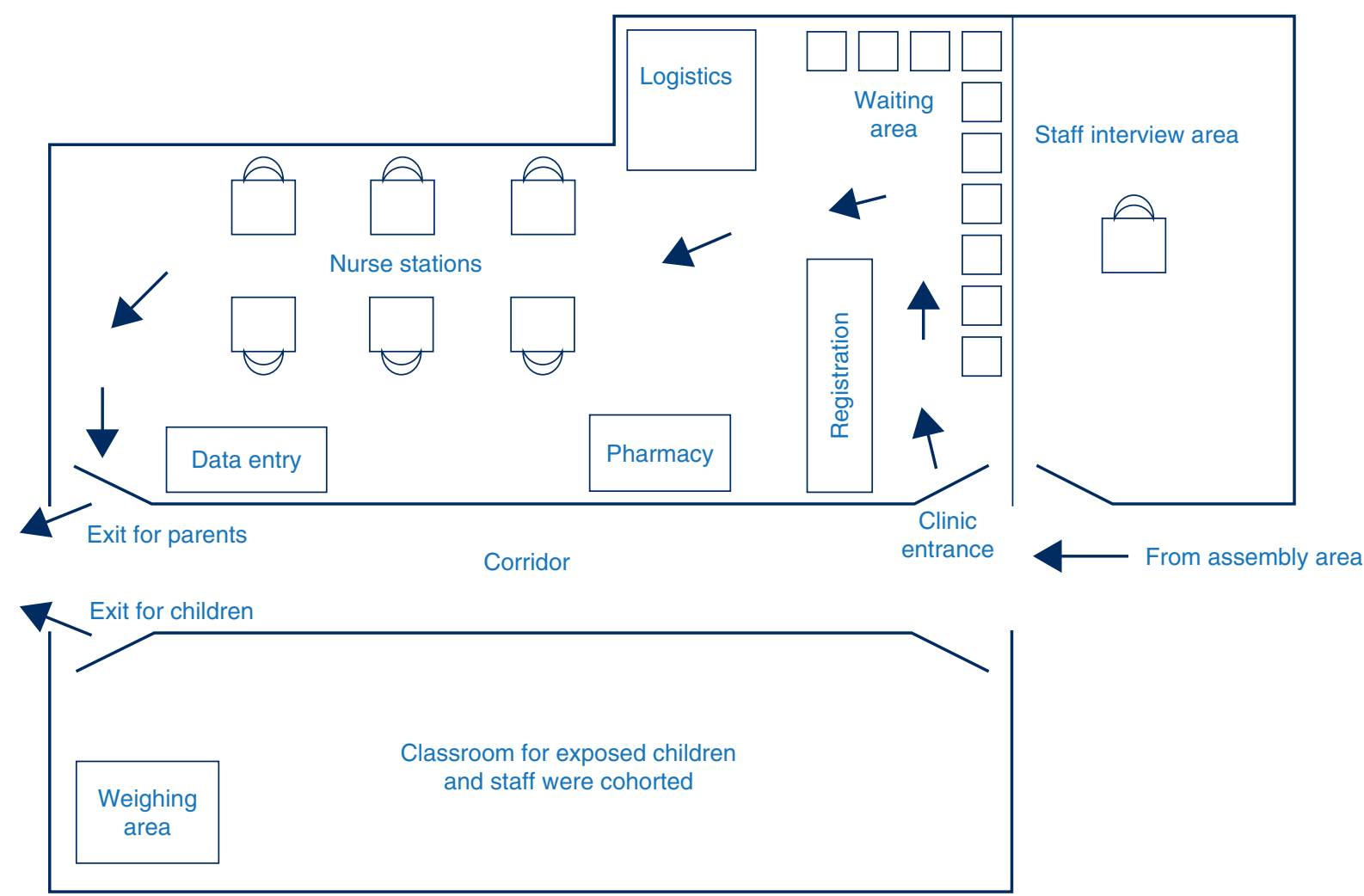

Figure 1. Layout and flow of people through the pandemic H1N1 2009 influenza prophylaxis clinic held in a school located in inner western Sydney, June 2009.

staff were assigned roles in control, operations, logistics and planning.

At $2.30 \mathrm{pm}$ a positive result for pandemic (H1N1) 2009 influenza was returned and the clinic became operational.

Family members arrived between $3 \mathrm{pm}$ and $5 \mathrm{pm}$ and gathered in the school assembly area, away from the clinic. Small groups were directed to the clinic by school staff. In order to prevent overcrowding in the clinic area, each group was limited to approximately 10 people.

The clinic was led by a clinic commander who addressed families at three separate group sessions during the afternoon. Staff from the school and district education office and some parents had been briefed earlier in the day, and the children and teachers in the affected class were also addressed as a group. At each briefing, information was provided and people were invited to ask questions.

The clinic was set up in a large classroom with an entrance at one end and an exit at the other (Figure 1). The parent(s) and other family members entered, were registered and then seated until a nurse was available to interview them. There were six stations, each staffed by one nurse equipped with hand-gel, tissues, information sheets and consent forms.
Infection control during the operation of the clinic was a major issue. Children and staff members in the same class as the case could have been infectious on the day of the clinic. The children were cohorted with their teachers in their classroom during the day and their outdoor playtime was allocated to a time when other schoolchildren and staff were in class.

In order to prevent clinic staff from being exposed to these children, the clinic was organised so that the parent was interviewed, consent was obtained and the parent given antiviral prophylaxis for the child while the other children remained in their classroom supervised by their teachers. The parents then left the clinic, collected their child and left the premises immediately. In this way, exposed children were not brought into the clinic area and most clinic staff were not required to wear personal protective equipment (PPE). Exposed children and staff were then excluded from school for 72 hours.

The presence of a pharmacist assisted clinical staff in efficiently ensuring small children (weighing less than $40 \mathrm{~kg}$ ) received correctly dispensed suspension. ${ }^{2}$ Each child was weighed in their classroom by a clinic staff member wearing personal protective equipment. The child's weight was noted on the registration form and antiviral medication for that child was dispensed to the parent. 
Exposed school staff members were interviewed in a separate room by a doctor wearing personal protective equipment. Information was provided, consent was obtained and the staff members were given antiviral prophylaxis in tablet form. They then went home or returned to supervise the children from the class.

\section{Outcome and discussion}

Seventy-four exposed children and all exposed staff $(n=9)$ were given antiviral prophylaxis at the clinic. One child was already symptomatic and had presented to $\mathrm{CHW}$ on the day of the clinic where he was given oseltamivir. Two of the 74 exposed children who had been given oseltamivir developed symptoms the next day and subsequently tested positive for pandemic (H1N1) 2009 influenza. These children were likely to be incubating the disease and to be infectious on the day of the clinic, highlighting both the timeliness of the clinic in terms of providing prophylaxis and the importance of maintaining good infection control when operating a clinic, in particular the cohorting of potentially infectious children away from clinic staff, school staff and other children.

The development of disease in the classmates suggests that transmission of the pandemic (H1N1) 2009 influenza virus had already occurred within the classroom. The advice to families to watch for symptoms, in conjunction with the antiviral intervention and exclusion, are likely to have reduced the risk of more widespread transmission of the virus among the school and surrounding community.

Feedback from the school and families was very positive. There was heightened community concern about pandemic (H1N1) 2009 influenza at the time. The group information sessions provided by the clinic commander were well received and ensured that anxiety was reduced and that the clinic operated in a calm and orderly manner. The Incident Command System approach to management of the clinic was appropriate and worked well, particularly as most SWAHS staff had previous experience or training in the Incident Command System.
The decision to exclude affected cases and provide prophylaxis to the affected students and staff allowed the rest of the school to remain open and was likely to have prevented further cases of influenza with the school and its community.

\section{Conclusion}

This paper describes a successful public health clinic providing prophylaxis to exposed students and staff during the CONTAIN phase of the pandemic (H1N1) 2009 influenza outbreak in NSW, Australia. Key elements of the success of the clinic included implementation of infection control measures, and isolation of cases and quarantining of close contacts, who may have been infectious; the availability of a pharmacist to assist in accurately dispensing suspension medication; deployment of experienced public health staff at short notice, and provision of accurate information to staff, school children and families attending the clinic.

\section{Acknowledgments}

The authors acknowledge the assistance of the Sydney West Area Health Service, the Centre for Health Protection at the NSW Department of Health, staff at the Children's Hospital at Westmead, the school and the district education office.

\section{References}

1. Commonwealth of Australia. Australian Health Management Plan for Pandemic Influenza. Commonwealth of Australia: Canberra; 2008.

2. Australian Government Department of Health and Ageing. Antiviral recommendations. Available from: http://www. healthemergency.gov.au/internet/healthemergency/ publishing.nsf/Content/clinical-antiviral (Cited 13 October 2009.)

3. World Health Organisation. Human infection with new influenza A (H1N1) virus: WHO consultation on suspension of classes and restriction of mass gatherings to mitigate the impact of epidemics caused by influenza A (H1N1), May 2009. Wkly Epidemiol Rec 2009; 84(27): 269-71.

4. US Department of Homeland Security. National Incident Management System. US Department of Homeland Security: USA; 2008. 